\title{
Wenn die Industrie ihre Beziehungen zur Ärzteschaft bekannt macht
}

\section{Hermann Amstad ${ }^{a}$,}

Walter H. Reinhart ${ }^{b}$

a Dr. med., Generalsekretär SAMW, Basel

b Prof. Dr. med., ehem. Chefarzt Innere Medizin, Kantonsspital Graubünden, Chur, und Präsident der «Beratenden Kommission»
Korrespondenz:

Schweizerische Akademie der Medizinischen

Wissenschaften SAMW

Petersplatz 13

CH-4051 Basel

mail[at]samw.ch
Bald 15 Jahre ist es her, dass die Schweizerische Akademie der Medizinischen Wissenschaften (SAMW) die Zusammenarbeit der Ärzteschaft mit der Industrie zum ersten Mal intensiv diskutiert hat. An einem Workshop am Sitz der Akademie in Basel referierten in- und ausländische Experten zu diesem heiklen Thema. In der Folge beauftragte der SAMW-Vorstand zwei Arbeitsgruppen mit der Ausarbeitung von Empfehlungen zu den Bereichen «Klinische Forschung» und «Weiter- und Fortbildung». Die Ärzteschaft nahm diese Empfehlungen bei deren Erscheinen 2002 mit höflichem Desinteresse zur Kenntnis.

Die Situation änderte sich schlagartig, als die SAMW 3 Jahre danach entschied, die Empfehlungen zu überarbeiten und - wichtiger noch - sie neu als Richtlinien zu veröffentlichen. Im Rahmen der Vernehmlassung trafen zahlreiche geharnischte Stellungnahmen ein, allerdings primär zum Kapitel «Weiter- und Fortbildung»: Auf Kritik stiessen namentlich die Regelungen, wonach zukünftig Monosponsoring von Fortbildungsanlässen nicht mehr zulässig und in der Regel für jeden Fortbildungsanlass eine Teilnahmegebühr zu entrichten sei. Diese Richtlinien flossen 2006 in die Standesordnung der FMH ein und sind seither für die Ärzteschaft bindend.

\section{«Beratende Kommission» hat Aufgaben erfüllt} Die SAMW setzte 2006 eine Beratende Kommission für die Umsetzung der Richtlinien «Zusammenarbeit Ärzteschaft-Industrie» ein. Diese begleitete seither die praktische Anwendung und Interpretation der Richtlinien. Zu Beginn waren Anfragen recht häufig, mit der Zeit nahmen diese ab.

Eine Anfrage im vergangenen Jahr ist von allgemeinem Interesse und soll hier kurz thematisiert werden: Gibt es von Seiten der SAMW Vorgaben betreffend des Sponsorings einer Fachgesellschaft oder einer der Fachgesellschaft gehörenden Stiftung? Dies ist in den gültigen SAMW-Richtlinien zwar nicht explizit geregelt; die Richtlinien machen aber diverse Vorgaben, die sich auch auf diese Situation anwenden lassen, nämlich:

- Sponsoring-Vereinbarungen werden schriftlich festgehalten; darin werden namentlich die gegenseitigen Pflichten und Verantwortlichkeiten geregelt.

- Es gibt mehrere voneinander unabhängige Sponsoren.

- Finanzielle Mittel aus dem Sponsoring werden auf ein dafür bestimmtes Konto der Stiftung bzw. Fachgesellschaft verbucht.

- Es gibt Transparenz über die eingenommenen und ausgeschütteten Beträge.
In der Praxis wurden bei den Richtlinien immer wieder Lücken und Präzisierungsbedarf festgestellt. Aus diesem Grund beschloss die SAMW 2012, diese zu revidieren. Dabei wurden neben der Überarbeitung und Ergänzungen verschiedener Abschnitte insbesondere ein neues Kapitel «Expertentätigkeit» hinzugefügt. Mit der Revision der Richtlinien sah die Beratende Kommission ihre Aufgaben als erfüllt an und beantragte deshalb ihre Auflösung. An seiner Sitzung von Ende November 2013 ist der SAMW-Senat diesem Antrag gefolgt und hat die Kommission unter Verdankung der geleisteten Dienste per 31. Dezember 2013 aufgelöst; die «Beratungsstelle» beim SAMW-Generalsekretariat ist jedoch weiterhin für die Beantwortung allfälliger Anfragen zuständig.

\section{Die Bedeutung des neuen} «Pharma-Kooperations-Kodex»

Der Auflösungsentscheid fiel dem Senat umso leichter, als mit dem Inkrafttreten des neuen Pharma-Kooperations-Kodex die Richtlinien von unerwarteter Seite Unterstützung erfahren haben. Im Jahr 2013 ist in den USA der «Physician Payment Sunshine Act» in Kraft getreten; er verpflichtet die Unternehmen, sämtliche Zahlungen an medizinische Leistungserbringer in einem öffentlich zugänglichen Register festzuhalten. Ähnliche gesetzliche Bestimmungen existieren auch in Frankreich sowie weiteren europäischen Ländern, und auch die EU signalisierte, solche Bestimmungen erlassen zu wollen. Dies veranlasste die European Federation of Pharmaceutical Industries and Associations (EFPIA), selbst aktiv zu werden und eigene Regelungen zu verabschieden (worauf die EU auf eigene Regelungen verzichtete). Da die EFPIAMitglieder verpflichtet sind, europäische Vorgaben national umzusetzen, haben scienceindustries, Interpharma und vips (Vereinigung Pharmafirmen in der Schweiz) vor kurzem den Pharma-Kooperations-Kodex verabschiedet. Gemäss diesem werden auch in der Schweiz ab 2015 alle Zahlungen an medizinische Leistungserbringer erfasst und im Folgejahr veröffentlicht. Damit wird jenes Leitmotiv aus den ACP-Guidelines von 1990, das sowohl die Empfehlungen als auch die Richtlinien stets begleitete, plötzlich Realität: «A useful criterion in determining acceptable activities and relationships is: would you be willing to have these arrangements generally known?» In der «Zusammenarbeit Ärzteschaft-Industrie» hat nun also die Letztere das Zepter übernommen und wird einen Schritt weitergehen. Die Ärzteschaft bekommt die Gelegenheit, sich darauf einzustellen und allenfalls gewisse Verhaltensmuster zu überdenken. 